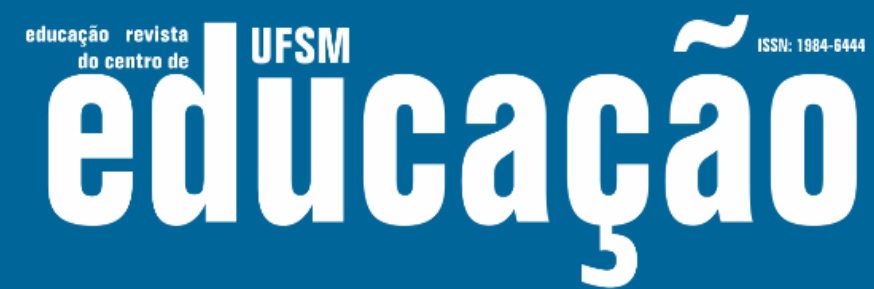

\section{Insegnare e apprendere la lingua seconda nel contesto migratorio. Il caso dell'Italia}

Teaching and learning a Second language, in a migration context: the Italian case

Ensinar e aprender a língua de acordo com o contexto migratório. $\mathrm{O}$ caso da Itália

Clara Silva

Università degli Studi di Firenze

Recebido em 03 de março de 2019

Aprovado em 09 de abril de 2019

Publicado em 10 de maio de 2019

\section{RIASSUNTO}

L'Italia è un paese tradizionalmente segnato da fenomeni di bilinguismo e plurilinguismo. L'attenzione su di essi è andata crescendo negli ultimi trent'anni, da quando l'aumento della presenza dei figli e delle figlie degli immigrati nella scuola ha fato emergere i loro bisogni specifici, compresi quelli relativi all'apprendimento della lingua italiana. II presente contributo ricostruisce a grandi linee i risultati degli studi di linguistica, mettendo a fuoco le coordinate generali entro cui si colloca la tematica dell'insegnamento e dell'apprendimento dell'italiano come lingua seconda. L'articolo si focalizza su alcuni nodi cruciali intorno a cui si sviluppa la pragmatica interculturale, tra cui l'interlingua, il transfer, la distanza tra prima e seconda lingua, i fattori psicosociali che interferiscono nell'apprendimento della lingua seconda. L'obiettivo è quello di mostrare come la questione dell'apprendimento della lingua del paese di immigrazione sia connesso non soltanto a fattori strettamente linguistici, ma anche biografici.

Parole chiave: Lingua seconda; Didattica; Figli e figlie degli immigrati.

\section{ABSTRACT}

Italy is traditionally marked by phenomena of bilingualism, and multilingualism. The attention on those aspects has been growing in the last thirty years, since the increase in the presence of children of immigrants in schools has revealed their specific needs, including those related to learn Italian. This paper gives a synthetic overview of the main outcome of linguistics studies, focusing on the general coordinates the topic of teaching and learning Italian, as a Second language, is placed whithin. The paper 


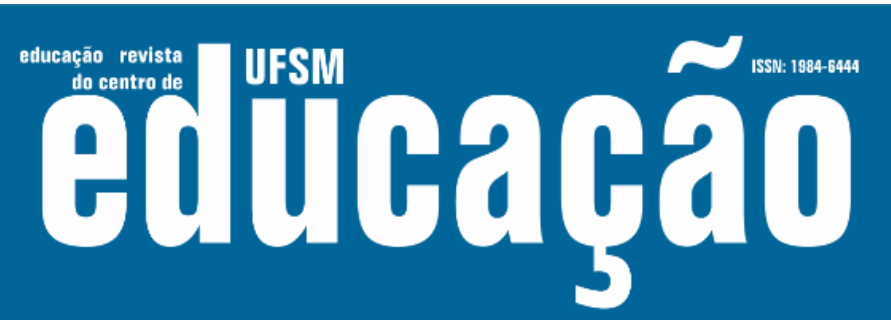

ISSN: 1984-6444 | http://dx.doi.org/10.5902/1984644437151

focuses on some crucial issues around which intercultural pragmatics develop including interlingua, transfer, and the gap between First and Second language, and psychosocial factors -, which affect Second language acquisition. Our aim is therefore to show how the topic of learning the language of the host country is connected not only to strictly linguistic, but also biographical, factors.

Keywords: Second Language; Didactics; Children of Immigrants.

\section{RESUMO}

A Itália é um país tradicionalmente marcado por fenômenos de bilinguismo e plurilinguismo. A atenção sobre eles vem crescendo nos últimos trinta anos, uma vez que o aumento da presença de filhos e filhas de imigrantes na escola tem revelado suas necessidades específicas, incluindo aquelas relacionadas ao aprendizado da língua italiana. O presente artigo reconstrói, grosso modo, os resultados dos estudos lingüísticos, focalizando as coordenadas gerais dentro das quais o tema do ensino e aprendizagem do italiano como segunda língua é colocado. O artigo enfoca algumas questões cruciais em torno das quais a pragmática intercultural se desenvolve, incluindo a interlíngua, a transferência, a distância entre a primeira e a segunda língua e os fatores psicossociais que interferem no aprendizado da segunda língua. $O$ objetivo é mostrar como a questão da aprendizagem da língua do país de imigração está ligada não apenas a fatores estritamente lingüísticos, mas também biográficos.

Palavras-chave: Segunda Língua; didática; filhos e filhas de imigrantes.

\section{Premessa}

La scuola italiana, malgrado le indicazioni contenute nella Costituzione e nelle disposizioni ministeriali, ha storicamente riservato scarsa attenzione alle questioni del bilinguismo o plurilinguismo degli allievi, salvo in alcune aree ove è impartita un'educazione plurilingue. Esse sono sostanzialmente tre: la Val d'Aosta (dove è parlato un idioma franco-provenzale e l'educazione bilingue avviene in francese), il Trentino-Alto Adige (dove nella provincia di Bolzano si parlano il tedesco e il ladino e dove l'insegnamento e l'apprendimento avvengono in lingua italiana, in lingua tedesca o in scuole trilingui) e il Friuli Venezia Giulia (dove nelle provincie di Trieste e di Gorizia esistono scuole statali con lingua d'insegnamento slovena). Iniziative isolate e talora non continuative sono state prese a livello regionale e locale anche in alcune regioni meridionali (PIZZORUSSO, 1983; FRANCESCATO, 1993) e, più di recente, attarverso azioni che mettono in rete realtà diverse sul territorio, come il progetto «Le piccole stelle del Carro Minore», che ha posto in collegamento le minoranze walser, friulana, albanese ed ellenofona (DONADA, 2006). 


\section{Aปtนดลดูão}

ISSN: 1984-6444 | http://dx.doi.org/10.5902/1984644437151

Allargando lo sguardo, vediamo che il fenomeno del bilinguismo e del plurilinguismo è in realtà ben più diffuso di quanto non appaia, esistendo infatti ben dodici idiomi che meritano la tutela prevista dall'articolo 6 della Costituzione italiana (DE MAURO, 1983, p. 5), in quanto parlati dalle minoranze sparse sul territorio nazionale: gli arbëresh/albanesi, i catalani, i croati, gli ellenofoni, i francofoni (francoprovenzali e occitani), i friulani, i germanofoni (carinziani, cimbri, mocheni, tedeschi e walser), i ladini, i sardi, gli sloveni (MORELLI, 2006, p. 8-12). Come si evince da questo elenco, le minoranze linguistiche alloglotte sarebbero dunque almeno una quindicina (FRANCESCATO, 1993, p. 314). Nel 1999 la tutela delle minoranze è stata regolamentata dalla Legge-quadro n. 482, seguita dal relativo regolamento di attuazione, con l'obiettivo di renderne omogenea la tutela. L'approvazione di questa normativa rappresenta "il punto di arrivo di una complessa evoluzione che ha portato al progressivo rovesciamento dell'indirizzo che, in materia di politica linguistica, era stato seguito dallo Stato italiano sin dall'epoca della sua Costituzione" (MORELLI, 2006, p. 7). La legge-quadro fornisce una cornice a un congiunto di leggi regionali che hanno cominciato ad essere promulgate a partire dagli anni '90 del secolo scorso, dal Veneto alla Sicilia, cui è seguita, nel 2003, una specifica normativa regionale per la Calabria (MORELLI, 2006, p. 12-13). Malgrado tale sforzo, il bilinguismo esistente in vari contesti, in particolare quello connesso alla presenza dei cosiddetti dialetti, che non sono varianti dell'italiano, ma lingue a sé (tranne che per i dialetti tosco-umbrolaziali), non è fatto oggetto di alcuna forma di protezione (BALBONI, 2002, p. 223).

La scuola ha molto spesso trascurato l'esistenza di un numero considerevole di allievi italiani che al loro ingresso nelle strutture educative non padroneggiano la lingua italiana ma solo il dialetto regionale (per lo più appartenenti alle classi sociali medioinferiori). Così come ha trascurato il fatto che i figli degli immigrati interni, varcando la soglia della classe, portavano con sé in molti casi il dialetto regionale dei luoghi di provenienza dei loro genitori (SOBRERO, 1973). Soltanto di recente, sulla scia della legislazione citata, che prevede l'uso della lingua minoritaria fin dalla scuola dell'infanzia, sono stati avviati progetti didattici con fondi del Ministero della Pubblica Istruzione e della Unione Europea al fine di sostenere il plurilinguismo nella scuola (MORELLI, 2006, p. 20). 


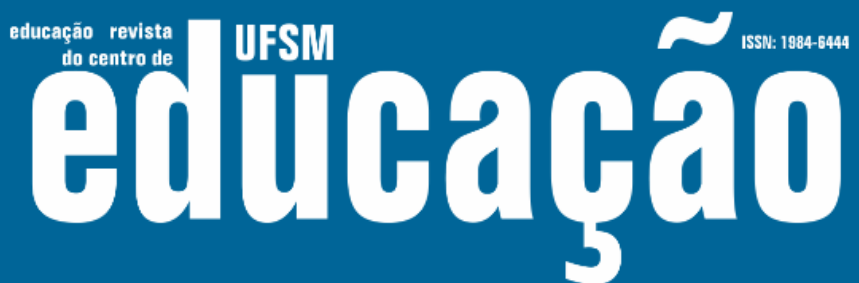

ISSN: 1984-6444 | http://dx.doi.org/10.5902/1984644437151

Questa prolungata disattenzione verso il pluralismo linguistico in Italia è testimoniata dal faticoso recupero che nel secondo ' 900 la filologia e la critica letteraria hanno operato del plurilinguismo italiano. In questo contesto la valorizzazione della linea dantesca, la poesia dialettale e autori come Gadda (si pensi al contributo di Gianfranco Contini a proposito) si è contrapposta al purismo di ascendenza petrarchesca e manzoniana. Negli ultimi decenni sono stati compiuti dei passi in avanti sia sul piano dei contenuti impartiti a scuola (inserimento di autori rappresentanti di linee linguistiche 'minoritarie') sia sul piano dell'attenzione agli aspetti psico-sociali dell'apprendimento linguistico.

$\grave{E}$ in questo quadro, di maggior attenzione al plurilinguismo, che s'inserisce la questione del riconoscimento delle esigenze specifiche poste dalla crescente presenza nella scuola dei figli degli immigrati di lingua nativa non italiana. Si tratta di una presenza linguisticamente molto varia, dal momento che gli immigrati in Italia provengono da un ventaglio assai variegato di paesi, a loro volta caratterizzati spesso da un pluralismo linguistico: si registrano infatti quasi 200 nazioni diverse di origine (CENTRO STUDI E RICERCHE IDOS, 2018, p. 9).

Alla luce di quanto esposto non è pertanto esatto attribuire all'arrivo dei figli degli immigrati nelle scuole la trasformazione dell'Italia in un paese plurilingue; semmai si dovrebbe dire che con il radicamento degli immigrati nella società italiana la questione del bilinguismo e del plurilinguismo è diventata più complessa, oltre che variegata. Un quadro oggi più complesso rispetto a quanto non fosse circa venti-venticinque anni fa, con il progressivo aumentare delle cosiddette seconde generazioni, cioè degli allievi figli di immigrati nati e cresciuti in Italia, i quali, allorché frequentano i servizi educativi per la prima infanzia, sono esposti alla lingua italiana fin da piccoli (FAVARO, 2011). La ricchezza del plurilinguismo, tuttavia, continua ancora essere vissuta nella scuola come un problema, in quanto sono ancora insufficienti gli strumenti di accoglienza e di inclusione linguistica efficaci per gestire classi linguisticamente e culturalmente eterogenee (SILVA, 2005).

La questione dell'apprendimento e dell'insegnamento della lingua seconda per gli allievi dell'immigrazione deve essere affrontata con maggior consapevolezza, attingendo teorie e metodi dagli studi specifici in materia e dalle proposte didattiche 


\section{Aปtนดลดูão}

ISSN: 1984-6444 | http://dx.doi.org/10.5902/1984644437151

maturate sulla base di esperienze portate avanti talora per anni. Allo stesso tempo è necessario un maggior riconoscimento della figura dell'insegnante della lingua seconda. Sono invece a disposizione degli insegnanti nelle varie discipline sussidi didattici molto utili, messi a punto da associazioni che da anni lavorano nel settore, come il Centro Come di Milano, o da centri universitari specializzati sull'insegnamento dell'italiano come lingua straniera che, in seguito al radicamento dell'immigrazione nel territorio, si sono occupati anche dell'apprendimento/insegnamento dell'italiano come lingua seconda. In centri del genere da anni ha luogo la formazione degli insegnanti di italiano come lingua seconda, i quali ottengono una specifica certificazione (cfr. il progetto Alias presso il Laboratorio Itals dell'Università di Venezia oppure il Centro Certificazione CILS dell'Università per Stranieri di Siena, sui quali si vedano, rispettivamente, https://www.itals.it/alias e BARNI, VILLARINI, 2001).

\section{Dalla prima alla seconda língua}

Per una didattica efficace della lingua seconda rivolta agli alunni dell'immigrazione è importante conoscere alcuni processi che presiedono all'apprendimento della L2 (detta anche "lingua d'arrivo"). Con questa espressione si intende la lingua appresa dopo la prima, cioè dopo quella materna o d'origine. In particolare, nell'ambito dell'insegnamento della lingua ad allievi stranieri o figli di immigrati, è la lingua appresa in un Paese dove essa costituisce la lingua parlata abitualmente e, in questo caso, si contrappone a lingua straniera (ad esempio: un allievo figlio di immigrati di madrelingua cinese impara nella scuola italiana l'italiano come L2, mentre sempre nella scuola impara l'inglese come lingua straniera). Con "lingua seconda" ci si riferisce a qualsiasi lingua appresa dopo la prima, e cioè anche alle terze, quarte lingue ecc. (LUISE, 2006).

Le ricerche su come si apprende una lingua seconda sono relativamente recenti. II primo e il più importante filone di ricerca si colloca all'interno del paradigma comportamentistico (che attribuisce un'importanza centrale nel processo di apprendimento alla formazione di abitudini) ed è l'“analisi contrastiva", in cui il sistema linguistico di partenza (lingua madre o lingua prima) e quello di arrivo (lingua seconda) 


\section{Eีtดaดูão}

ISSN: 1984-6444 | http://dx.doi.org/10.5902/1984644437151

degli apprendenti sono messi a confronto e a partire da questo confronto sono avanzate previsioni sulle aree in cui gli apprendenti possono incontrare maggiori difficoltà (PALLOTTI, 1998, p. 17).

Successivamente, altre ricerche hanno chiarito che gli errori degli apprendenti in molti casi non possono essere spiegati semplicemente come interferenze tra la prima e la seconda lingua. L'orizzonte teorico di riferimento di queste nuove ricerche è quello cognitivista, secondo il quale colui che apprende una lingua non interiorizza abitudini, ma scopre regole grammaticali attraverso un ragionamento creativo. In altre parole l'apprendente, guidato da un dispositivo innato (la "Grammatica Universale" di Chomsky), formula regole sulla lingua e produce costruzioni creative che non possono essere ricondotte a un semplice condizionamento da parte degli input.

Nascono così gli studi di "analisi degli errori", che si fondano sul presupposto secondo il quale commettere errori è per l'apprendente un modo di mettere alla prova le proprie ipotesi sulla natura della lingua che sta imparando. In altri termini l'apprendente possiede una dotazione innata di strategie con sui ricostruire il nuovo codice linguístico: "gli errori devono essere spiegati e previsti a partire da queste strategie e non mettendo semplicemente a confronto i sistemi linguistici di partenza e di arrivo" (PALLOTTI, 1998, p. 59).

Nella nuova prospettiva, fondamentali risultano allora gli studi sulle "sequenze evolutive" dell'apprendimento della seconda lingua. Tali studi hanno provato che "apprendenti con retroterra linguistici diversi seguono tutti lo stesso percorso nell'acquisizione di certe strutture, un percorso che pare essere quindi il prodotto dall'interazione di due fattori principali: la struttura della lingua da apprendere e l'organizzazione della mente umana" (PALLOTTI, 1998, p. 59).

Diversi tra questi studi hanno fortemente ridimensionato il ruolo della prima lingua, che invece per i comportamentisti era ritenuto fondamentale, poiché secondo questi una serie di 'abitudini' dovevano essere superate affinché si instaurassero quelle della nuova lingua. In seguito gli studiosi hanno proposto una posizione per certi versi intermedia tra le due, in cui alla prima lingua è attribuita un'influenza di un certo rilievo sulle produzioni linguistiche via via elaborate dagli apprendenti. II rapporto tra sequenze evolutive universali e condizionamenti della prima lingua non è più 


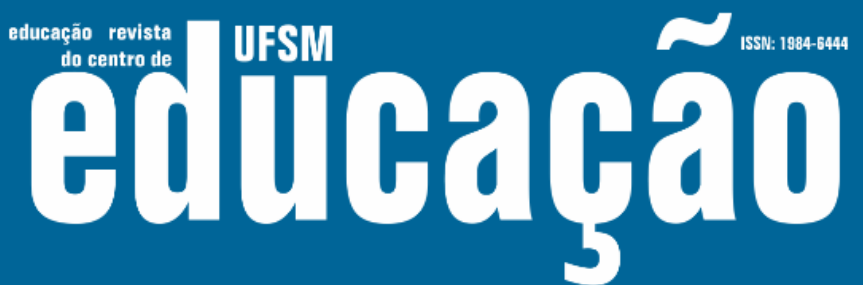

ISSN: 1984-6444 | http://dx.doi.org/10.5902/1984644437151

interpretato nei termini di un'alternativa. L'obiettivo è quello di riconoscere quali aspetti di una particolare varietà di seconda lingua in un apprendente vanno ricondotti a strategie universali e quali invece alla sua lingua materna.

\section{Dall'errore all'interlingua}

Nel quadro delle ricerche che abbiamo appena richiamato è stata introdotta la nozione di "interlíngua" come quella nozione capace di spiegare la dinamica dell'apprendimento di una lingua come seconda lingua o lingua d'arrivo. L'interlingua è un vero e proprio sistema linguistico e non un insieme caotico di errori; si tratta cioè di un sistema governato da regole, anche se quest'ultime non corrispondono se non in parte a quelle della lingua d'arrivo. Con questo concetto si supera dunque la concezione tradizionale che interpreta i vari stadi di apprendimento della seconda lingua come produzioni devianti viste solo in termini di errori. Tali stadi vengono così a diventare delle fasi ben precise di una strategia di apprendimento, il cui studio è utile per programmare la stessa didattica della lingua seconda (PALLOTTI, 1999, p. 54).

La ricerca si è concentrata in modo particolare sulle prima fasi dell'apprendimento della lingua seconda, rilevando che all'inizio l'interlingua degli apprendenti appare come una "lingua senza grammatica", dal momento che le prime strutture che vengono apprese sono parole isolate o formule non analizzate. Solo in un secondo momento viene appreso, per fasi, quell'insieme di regole e morfemi che compongono la grammatica di una lingua.

Le prime parole che vengono apprese sono legate soprattutto all'interazione e alle necessità comunicative primarie legate a questa. Essendo difficile poter condurre ricerche su adulti apprendenti la lingua seconda in situazioni di apprendimento naturali, le ricerche condotte sull'interlingua degli apprendenti l'italiano sono state effettuate per lo più studiando bambini inseriti in istituzioni scolastiche. Da esse risulta che le prime espressioni pronunciate svolgono determinate funzioni quali: a) sollecitare l'attenzione (es.: "guarda", "maestra", "bimbi", "ecco", “io", nomi propri); b) regolare l'interazione (es.: "aspetta”, "andiamo", "va via”, "aiuto”, "piano”, "ciao", "scusa”, "grazie”, "sì", "no”); c) riferirsi a oggetti/eventi (es.: "questo", "quello", "cosi”); d) descrivere/valutare (es.: 


\section{Aปtนดลดูão}

ISSN: 1984-6444 | http://dx.doi.org/10.5902/1984644437151

"bella", "grande”, "brava”, "brutto”, “mio”, "buono”, "pipi”); e) varie (es.: "uno”, "due”, "tre", "quattro"...).

Nelle prime fasi di apprendimento vengono apprese anche formule con cui gli apprendenti producono espressioni che paiono sorprendentemente corrette e ben più complesse della media dei loro enunciati. Un esempio può essere rappresentato dall'espressione "come si chiama?», che contiene la forma riflessiva del verbo e l'accordo per tempo e per numero (PALLOTTI, 1998, p. 23-26).

Proviamo qui a riepilogare i risultati delle ricerche sulle sequenze evolutive dell'interlingua in italiano, fondandoci soprattutto sugli studi di Pallotti e Giacalone Ramat (PALLOTTI, 1998, p. 49-59; GIACALONE RAMAT, 1993, p. 369-399).

Morfologia verbale: 1a fase) radice verbale o forma basica del verbo (es.: io mangia); infinito poi indicativo presente); $2 a$ fase) participio passato (dapprima per indicare azioni o eventi determinati e conclusi; es.: io arrivato); 3a fase) imperfetto (dapprima verbi stativi, cioè indicanti uno stato, o durativi - es.: poteva, era -, poi altri verbi); 4a fase) futuro, condizionale (poi congiuntivo).

Nelle prime fasi si osserva una generalizzazione delle desinenze verbali di seconda e terza persona singolare al presente, che vengono estese alle altre persone (es.: io va, io risponde, tu dice). Nella prima fase, dominata dalla forma basica, vengono impiegati avverbi per indicare il tempo; inoltre, soprattutto negli adulti, compaiono forme perifrastiche di tempi semplici non ancora acquisiti (es.: ero sono $=$ erano; sarà aiuto = aiuterà).

Inizialmente vi è un uso scarso o nullo della copula.

Per quanto concerne le modalità (asserzioni, dubbio, desiderio), dapprima restano implicite, poi sono espresse con perifrasi, con l'infinito, con avverbi (es.: non so, penso, credo, forse, magari, possibile, volere, potere, dovere, bisogna) e infine con i tempi modali (congiuntivo e condizionale; a partire da vorrei e sarebbe).

Morfologia nominale: a) prima compare la categoria di numero poi quella di genere (il morfema del plurale maschile -i viene usato più frequentemente $e$ correttamente dei morfemi del genere maschile e femminile singolare); in questa fase, rispetto al genere, i nomi sono unità opache non analizzate; es.: uomo; b) viene introdotto l'articolo definito/indefinito (le parole vengono combinate tra loro in enunciati 


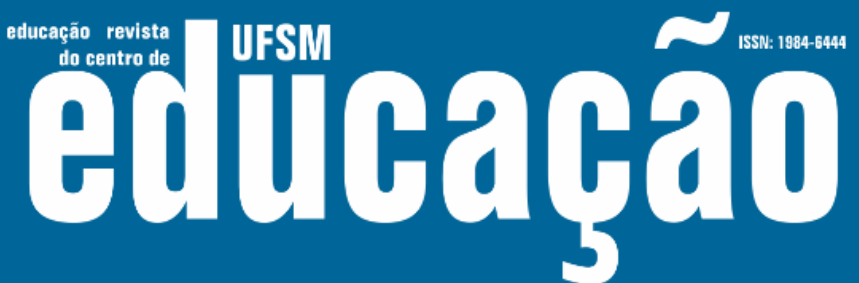

ISSN: 1984-6444 | http://dx.doi.org/10.5902/1984644437151

e sintagmi; es.: la maestra); c) il nome viene accordato con l'aggettivo attributivo (es.: acqua calda); d) l'accordo viene esteso all'aggettivo predicativo (es.: la cucina è piccola); e) viene introdotto l'accordo tra il nome e il participio passato (es.: siamo partite). Nelle fasi più avanzate il controllo del nome si estende da elementi sintatticamente più vicini (sintagma nominale) a elementi più distanti (sintagma verbale); es.: le mie care bambine sono tornate. Già nella prima fase compare l'opposizione lui/lei, di grande importanza a livello pragmatico. Precocemente compaiono anche i pronomi io e $t u$, riferiti ai protagonisti dell'enunciazione. A fianco del riferimento deittico (in cui ci si riferisce a qualcuno, ad esempio indicandolo) compare molto presto il riferimento anaforico (in cui la persona a cui si fa riferimento non è presente) - tramite la terza persona - a entità precedentemente introdotte nel discorso. Tuttavia si osserva una sovraestensione delle forme usate con funzioni di soggetto al posto di quelle per l'oggetto, così come l'acquisizione più tarda delle forme del plurale. L'espressione della persona ha una evidente rilevanza sul piano pragmatico, specialmente nelle interlingue che non presentano ancora una morfologia verbale sviluppata; ciò spiega "perché compaiono frequentemente pronomi personali soggetto nelle interlingue italiane, mentre nell'italiano non esprime il soggetto pronominale, salvo casi di enfasi o di contrasto" (GIACALONE RAMAT, 1993, p. 387).

Sintassi: nelle prime fasi dell'interlingua si osserva un ordine delle parole soggetto a interferenze (es.: "cielo di macchina" invece di "macchina di cielo", per "aereo", presso apprendenti cinesi); per quanto concerne la combinazione di frasi, queste vengono giustapposte o collegate paratatticamente; compaiono forme di subordinazione tra proposizioni mediante avverbi nel caso di relazioni temporali, causali, finali, condizionali, consecutive (es.: mi sono fermato al bar, poi sono salito a casa); da ultimo compaiono forme di subordinazione tra proposizioni attraverso completivi nel caso di relazioni relative, oggettive, soggettive (mentre in una prima fase sono per lo più assenti le forme di congiunzione tra le proposizioni). Alcuni studi mostrano però che fin dalle fasi iniziali, seppure con frequenza bassa, compaiono con uso appropriato congiunzioni come che, quando, perché, se.

Per quanto riguarda la negazione, nelle prime fasi dell'interlingua essa è espressa mediante una particella invariabile (es.: no io freddo; no mangia; no cosi). 


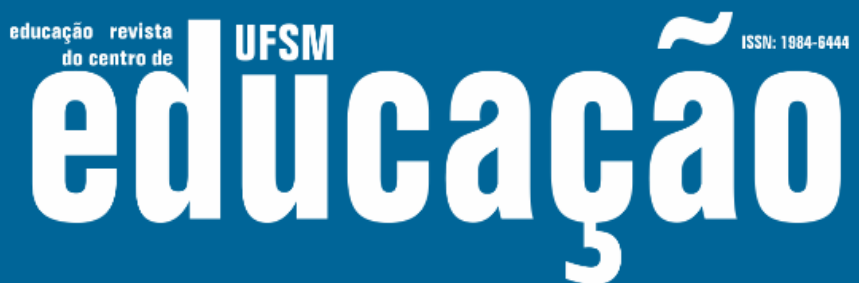

ISSN: 1984-6444 | http://dx.doi.org/10.5902/1984644437151

Sempre nelle prime fase, le preposizioni (come avviene per gli articoli) mancano o sono usate limitatamente; vi è poi un uso di funtori grammaticali in contesti e con funzioni non standard (uso abbondante e a sproposito del di e di chi/che).

Circa la fonologia, essa è caratterizzata, come per qualsiasi apprendimento di una lingua seconda, da fenomeni di interferenza della prima lingua (come si vedrà meglio nel paragrafo seguente). La ricerca, in questo settore, è molto recente, e sono tuttora pressoché assenti applicazioni didattiche (BROSELOW, KANG, 2013, p. 552; SISINNI, 2016).

Per quanto riguarda il lessico, già si è detto qualcosa a proposito delle prime parole, tra cui compaiono forme di negazione, forme di saluto e di commiato, di ringraziamento e altre espressioni frequenti, tra cui formule non analizzate. Nelle interlingue iniziali non sempre è chiara la distinzione in classi di parole e determinati elementi lessicali possono essere tanto nomi quanto verbi, l'arricchimento del vocabolario dipende dalle attività e dai contesti con sui l'apprendente ha a che fare, il lessico è più semplice e meno preciso della lingua d'arrivo.

Più in generale, nelle interlingue dell'italiano si è notato che la morfologia risulta semplificata e risponde a criteri di trasparenza. Esempi di semplificazione sono la sovraestensione di aggettivi e participi al maschile singolare (es.: siamo andato, suo moglie), la generalizzazione ad altre persone di desinenze verbali di seconda e terza persona singolare presente (vedi sopra: morfologia verbale). Esempi di trasparenza sono le cosiddette flessioni analogiche (es.: io dicio, io conoscio, puliscio, romputo, ovI) miranti a rendere regolari i paradigmi e presenti anche nelle varietà infantili degli italofoni, il mantenimento di forme piene (es.: de la, in la, questo anno), le forme perifrastiche di tempi semplici non acquisiti (vedi sopra: morfologia verbale). Rispetto alla questione della semplificazione di una struttura, si deve osservare che essa può preludere o all'apprendimento della tappa successiva, cui ci si prepara proprio evitando l'accumularsi della complessità, o alla fossilizzazione, che permette l'uso di una competenza linguistica interiorizzata (in questo caso l'operazione sarà evitata ogni volta che ciò sarà possibile).

\section{II transfer e la distanza tipologica tra L1 e L2}




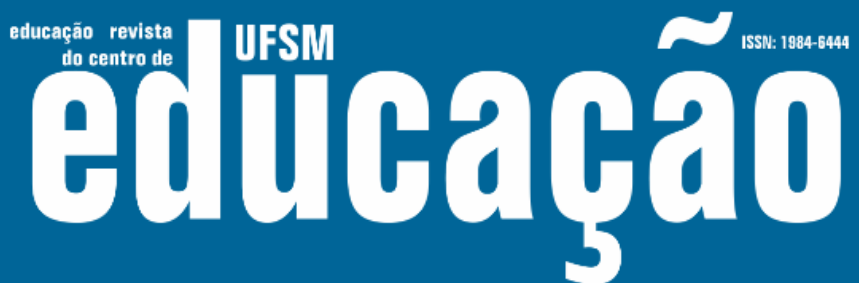

ISSN: 1984-6444 | http://dx.doi.org/10.5902/1984644437151

Come si è detto più sopra, a fianco di strategie universali che segnano il percorso di acquisizione di certe strutture linguistiche indipendentemente dalla lingua d'origine vi sono dei condizionamenti della prima lingua sull'interlingua, ovvero nell'apprendimento della lingua seconda.

Tali condizionamenti sono definiti come "transfer". Con questa espressione ci si riferisce all'influenza della lingua prima o di altre lingue apprese in precedenza sull'apprendimento della lingua seconda. II concetto di transfer ha sostituito quello di interferenza, proprio perché si riferisce a un fenomeno non soltanto negativo e che non si limita al trasferimento di strutture da una lingua all'altra. Alcuni ricercatori giungono fino a ribaltare l'idea tradizionale per cui le abitudini linguistiche della prima lingua devono essere sostituite dalle abitudini nuove e diverse della seconda, sostenendo invece che la lingua materna ha un ruolo di facilitatore nell'apprendimento della lingua seconda (il sistema di quest'ultima viene ricostruito gradualmente facendo riferimento a quello della prima).

Il transfer avviene a tutti i livelli del linguaggio, da quello della fonologia (cioè della pronuncia delle singole parole), a quello dei fenomeni prosodici e paralinguistici (cioè della produzione di frasi, relativamente all'intonazione, al ritmo, all'accentuazione), da quello del lessico (cioè dei significati delle parole e della corrispondenza tra significante e significato) a quello della sintassi (la disposizione degli elementi nella costruzione del periodo), da quello della morfologia (trasferimento di morfemi propri della lingua seconda laddove non sono richiesti in analogia con quanto avviene nella lingua prima) fino a quello del discorso (il modo in cui si collegano gli enunciati).

Rispetto al tema dell'insegnamento dell'italiano come lingua seconda è dunque importante tener conto della complessità di questi fenomeni. Si tratta allora di considerare il profilo linguistico di partenza degli apprendenti. Una certa conoscenza della struttura della lingua prima o delle lingue prime dell'apprendente può risultare utile all'insegnante per riconoscere quali sono le aree di transfer negativo e quelle di transfer positivo, ovvero le strutture linguistiche simili e quelle divergenti tra le due lingue. Rispetto a quest'ultimo caso, a livello fonologico, ad esempio, un arabofono fa fatica a distinguere tra /o/ e /u/ e tra /e/ e /i/ perché in arabo esistono solo tre vocali, 


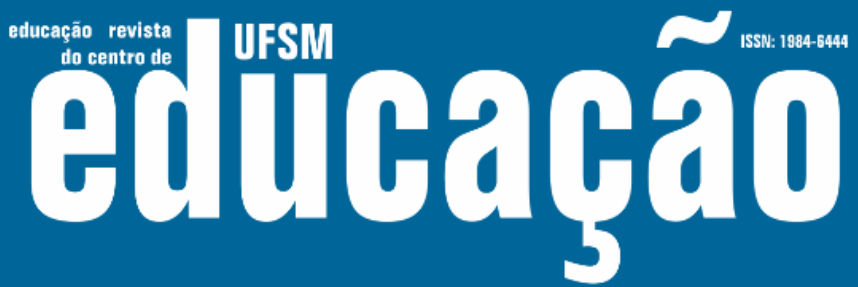

ISSN: 1984-6444 | http://dx.doi.org/10.5902/1984644437151

/a/, /i/ e /u/ (PALLOTTI,1998, p. 60-63). Nel caso di apprendenti di origine capoverdiana, la casistica è varia e riflette la complessità della situazione linguistica di Capo Verde. Qui infatti la lingua di uso quotidiano è il creolo capoverdiano (nato dall'incontro tra idiomi parlati sule coste africane e lingue europee, in special modo il portoghese), ma ad esse si affianca il portoghese, lingua ufficiale utilizzata nei documenti e nelle comunicazioni scritte e in prevalenza nei mass media. Nella scuola copoverdiana fino al 1975, quando il paese ha raggiunto l'indipendenza, era utilizzato esclusivamente il portoghese. Da allora in poi è stato introdotto anche il creolo, per ora solo nel parlato. Per il capoverdiano apprendente il portoghese nel luogo d'origine esiste dunque un fenomeno di transfer dal creolo al portoghese, fenomeno su cui recentemente si è cominciato a riflettere allorché sono stati rilevati insuccessi scolastici derivanti da carenze linguistiche in portoghese. Questa situazione già di per sé complessa non può che influire sull'apprendimento della lingua d'arrivo nel caso di una migrazione. Situazioni più o meno analoghe accadono per apprendenti provenienti da altri paesi, come il Marocco (dove può avvenire transfer dall'arabo marocchino all'arabo classico, studiato a scuola), la Cina (dove lo stesso fenomeno può avvenire dal dialetto regionale al mandarino) o anche le Filippine (dove il tagalog può essere insieme lingua madre e lingua ufficiale oppure soltanto la seconda). Naturalmente per chi nasce in Italia la situazione può essere diversa e dipende dalla lingua o dalle lingue utilizzate nella comunicazione interfamiliare.

La variazione nell'apprendimento della seconda lingua dipende anche in parte dalla "distanza" fra le due lingue che vengono a contatto nella mente dell'apprendente. I linguisti chiamano questa distanza "tipológica" e sostengono che apprendenti che hanno una prima lingua tipologicamente (e geneticamente) prossima allitaliano (francese, spagnolo, portoghese o creoli su base francese e portoghese) hanno una maggior facilità di apprendimento dell'italiano rispetto a parlanti che abbiano una prima lingua tipologicamente (e geneticamente) lontana dall'italiano, come il tamil, il cinese, il tagalog, il tigrino, l'arabo, o le lingue diffuse nell'Africa sub sahariana (BANFI, 1993, p. 37). Anna Giacalone Ramat porta il caso dell'apprendente cinese, la cui prima lingua d'arrivo, l'italiano, ha un alto livello di morfologizzazione (manifestato in particolare da suffissi e desinenze portatori di distinzioni di genere, numero, persona, tempo ecc.). 


\section{Elthapão}

ISSN: 1984-6444 | http://dx.doi.org/10.5902/1984644437151

Questo apprendente "dovrà imparare a segnalare sul nome e sul verbo categorie grammaticali che nella sua L1 non hanno codificazione linguistica analoga o mancano di una codificazione linguistica esplicita: nel cinese, ad esempio, il ruolo del contesto e di elementi avverbiali nella segnalazione di relazioni temporali di passato è maggiore che nelle lingue europee" (GIACALAONE RAMAT, 1993, p. 363). Sempre secondo Giacalone Ramat si possono fare delle ipotesi di natura psicolinguistica a riguardo, nel senso che, a fianco della distanza per così dire oggettiva di cui si è appena detto, se ne pone anche una legata alla percezione soggettiva dell'apprendente, che formula delle ipotesi intorno al rapporto contrastivo tra la lingua di partenza e quella d'arrivo.

\section{Fattori psico-sociali e socio-culturali che intervengono nell'apprendimento dell'italiano come L2}

Altri fattori, di carattere psico-sociale e socio-culturale, intervengono nell'apprendimento della lingua seconda. Alcuni son legati al gruppo di provenienza dell'individuo e cioè a determinati caratteri di tale gruppo (la sua coesione interna, il suo approccio verso l'esterno, le sue dimensioni, la sua prossimità linguistica ecc.), altri all'individuo in sé (il suo progetto migratorio, la sua personalità, il suo stile cognitivo, il suo patrimonio linguistico-culturale ecc.), altri ancora agli atteggiamenti e alle risposte del contesto in cui avviene l'apprendimento (natura e qualità dei contatti dei nativi con i non nativi, stereotipi, pregiudizi, modalità di accoglienza, politiche di immigrazione, razzismo istituzionale ecc.). Giacalone Ramat osserva che nel caso dei cinesi può esservi una correlazione tra la configurazione sociale delle loro comunità in cui viene esercitata una forte funzione di sostegno e di controllo verso i propri membri e la cui unità primaria è il nucleo familiare - e lo scarso successo nell'apprendimento della seconda lingua. II fattore socioculturale sarebbe così prevalente sulla questione della distanza tipologica della lingua cinese rispetto a quella italiana, perché nel caso di altri apprendenti la cui prima lingua è altrettanto distante dall'italiano quanto il cinese, come nel caso di apprendenti di lingua materna wolof o tigrino, non si rilevano difficoltà di apprendimento di entità simile (GIACALONE RAMAT, 1993, p. 353). 


\section{Elthapão}

ISSN: 1984-6444 | http://dx.doi.org/10.5902/1984644437151

Vanno poi considerati elementi individuali di ordine biologico, quali l'età. Benché ci siano pareri contrastanti, nel complesso si è d'accordo nel ritenere che più un apprendente è giovane, maggiore è il suo vantaggio nel processo di apprendimento.

Un altro fattore da tenere in considerazione è la percezione del proprio processo di apprendimento, ovvero la riflessione metalinguistica dell'apprendente, che può agire come fattore di promozione verso lo sviluppo di interlingue standard e come vettore di integrazione.

\section{Per concludere: dalla teoria alla pragmatica interculturale}

L'attenzione al livello psico-sociale dell'apprendimento, ma anche a fattori che i linguisti definiscono di pragmatica interculturale, aiuta a leggere determinati fenomeni non soltanto sul piano strettamente linguistico di avvenuta o meno acquisizione di certe strutture della lingua seconda, ma anche come effetti di strategie più o meno consapevoli a livello pragmatico nello scambio e nell'interazione tra nativi e non nativi.

$\mathrm{Si}$ possono ad esempio interpretare in questa chiave certi fenomeni di fossilizzazione. In Italia tra i pochi studi condotti in questo ambito uno riguarda i filippini e rileva interlingue praticamente fossilizzate, malgrado una lunga permanenza in Italia e forme più o meno ampie di esposizione all'italiano (ORLETTI, 1988). Tale fenomeno può essere interpretato da molteplici prospettive. In una propspettiva sociologica lo si considera favorito dalla coesione del gruppo di appartenenza e da un suo relativo isolamento dalla società autoctona. In una propsettiva psicológica può essere invece ricondotto a una volontà inconscia di mantenere una certa barriera tra il proprio gruppo e le altre comunità con cui si entra in contatto (non solo i nativi, ma anche altri gruppi immigrati), mentre da una prospettiva linguistica può esser fatto derivare dalla relativa facilità di avvio della fase iniziale della costruzione dell'interlingua, sulla base di una affinità tra L1 e L2 (questo ad esempio è sottolineato nel caso dei gruppi ispanofoni che apprendono l'italiano nel contesto migratorio: FERRARIO, 2013, p. 335).

In un'ottica che considera le dinamche relazionali che si danno nei contesti migratori, certi fenomeni di transfer dalla lingua prima alla lingua seconda possono essere intrepretati come effetti di una strategia pragmatica funzionale all'interazione e 


\section{Aปtนดaดูão}

ISSN: 1984-6444 | http://dx.doi.org/10.5902/1984644437151

non tanto di carenze o deficit linguistici. Tali strategie potrebbero essere finalizzare a creare un buon rapporto con gli interlocutori nativi, suscitando cioè presso questi ultimi un atteggiamento di benevolenza, dal momento che si rinuncia a porsi su un piano di parità assoluta nella competenza linguistica. Ciò avviene sul piano fonologico o paralinguistico (accentuazione di parole o periodi, ritmo), ma tra le varie strategie pragmatiche messe in atto dai non nativi ve ne sono alcune inerenti al piano strettamente pragmatico, come le ammissioni di incompetenza (PALLOTTI, 1998, p. 148-149). Strategie come queste possono essere lette anche come risposte all'interiorizzazione di un'immagine stereotipata di certi parlanti non nativi presso i nativi. Mentre in taluni casi tale immagine non è negativa - ed è quello di parlanti una prima lingua riconosciuta come prestigiosa (francese, tedesco, inglese ecc.) - in altri siamo di fronte a stereotipi o pregiudizi che hanno forte incidenza negativa sulla relazione e gravi conseguenze sociali. Stereotipi e pregiudizi che entrano in gioco anche laddove tali strategie non vengono messe in atto dai parlanti non nativi e costituiscono una sorta di filtro nell'interazione con i nativi.

\section{Riferimenti bibliografici}

BALBONI, Paolo E. Le sfide di Babele: Insegnare le lingue nelle società complesse. Torino: UTET, 2002.

BANFI, Emanuele. Italiano come L2. In: BANFI, Emanuele (Org.). L'altra Europa linguistica. Scandicci: La Nuova Italia, 1993, p. 35-76.

BARNI, Monica; VILLARINI, Andrea (Org.). La questione della lingua per gli immigrati stranieri: Insegnare, valutare e certificare l'italiano L2. Milano: Franco Angeli, 2001.

BROSELOW, Ellen; KANG, Yoonjung. Phonology and speech. In: HERSCHENSOHN, Julia, YOUNG-SCHOLTEN, Martha (Org.). The Cambridge Handbook of Second Language Acquisition. Cambridge: Cambridge University Press, 2013, p. 529-553.

CENTRO STUDI E RICERCHE IDOS. Dossier Statistico Immigrazione: 2018. Roma: Centro Studi e Ricerche Idos, 2018.

DE MAURO, Tullio. L'educazione plurilingue nelle scuole italiane. In: DI IORIO, Francesca (Org.). L'educazione plurilingue in Italia. «I Quaderni di Villa Falconieri», 2. Frascati: CEDE, 1983, p. 1-7. 


\section{Elthapão}

ISSN: 1984-6444 | http://dx.doi.org/10.5902/1984644437151

DONADA, Pierino. II progetto «Le piccole stelle del Carro Minore». 4 minoranze in 4 realtà. In: Le minoranze linguistiche in Italia nella prospettiva dell'educazione plurilingue: La legge 482/1999 sulle minoranze linguistiche nel settore scolastico. (Numero monografico). Annali della Pubblica Istruzione, 5/6, 2006, p. 97-105.

FAVARO, Graziella. Una lingua "seconda e adotiva": l'italiano delle seconde generazioni. Italiano LinguaDue, 1, 2010, p. 1-14.

FERRARIO, Gloria. L'italiano degli immigrati ispanofoni: L'influenza della lingua1 nell'apprendimento di lingue affini. Italiano LinguaDue, 1, 2013, p. 314-340.

FRANCESCATO, Giuseppe. Sociolinguistica delle minoranze. In: SOBRERO, Alberto A. (Org.). Introduzione all'italiano contemporaneo: La variazione e gli usi. RomaBari: Laterza, 1991, p. 311-340.

GIACALONE RAMAT, Anna. Italiano di stranieri. In: SOBRERO, Alberto A. (Org.). Introduzione all'italiano contemporaneo: La variazione e gli usi. Roma-Bari: Laterza, 1991, p. 341-410.

LUISE, Maria Cecilia. Italiano come seconda lingua: Elementi di didattica. Torino: UTET, 2006.

MORELLI, Domenico. La diversità linguistica e culturale in Italia. In: Le minoranze linguistiche in Italia nella prospettiva dell'educazione plurilingue: La legge 482/1999 sulle minoranze linguistiche nel settore scolastico. (Numero monografico). Annali della Pubblica Istruzione, 5/6, 2006, p. 6-22.

ORLETTI, Franca. L'italiano dei filippini a Roma. In: GIACALONE RAMAT, Anna (Org.). L'italiano tra le altre lingue. Bologna: II Mulino, 1988, p. 143-159.

PALLOTTI, Gabriele. La seconda língua.Milano: Bompiani, 1998.

PALLOTTI, Gabriele. Acquisire e insegnare l'italiano: dai processi naturali agli interventi didattici. In: FAVARO, Graziella (Org.). Imparare I'italiano imparare in italiano: Alunni stranieri e apprendimento della seconda lingua. Milano: Gueruni e Associati, 1999, p. 47-90.

PIZZORUSSO, Alessandro. L'attuazione del principio costituzionale di tutela dei gruppi linguistici in Italia. In: DI IORIO, Francesca (Org.). L'educazione plurilingue in Italia. «I Quaderni di Villa Falconieri», 2. Frascati: CEDE, 1983, p. 25-34.

SILVA, Clara. L'educazione interculturale: modelli e percorsi. Nuova edizione aggiornata e integrata. Tirrenia: Edizioni Del Cerro, 2005.

SISINNI, Bianca. Fonetica e fonologia della seconda lingua: Teorie, metodi e prospettive per la didattica. Roma: Carocci, 2016. 


\section{Althatẫo \\ 3}

ISSN: 1984-6444 | http://dx.doi.org/10.5902/1984644437151

SOBRERO, Alberto A. II cambio linguistico nell'acculturazione dell'immigrato: nuovi problemi di glottodidattica. In: A.a.V.v. Bilinguismo e diglossia in Italia. Pisa: Pacini, 1973, p. 137-148.

\section{Correspondência}

Clara Silva - Professora doutora associada em Pedagogia Geral e Social do Departamento de Educação, Línguas Estrangeiras, Interculturalidade, Literaturas e Psicologia da Universidade de Florença.

Università degli Studi di Firenze - UniFI - P.zza S.Marco, 4 - 50121 Firenze Centralino +39 05527571 - E-mail: urp(AT)unifi.it - Posta certificata: ateneo(AT)pec.unifi.it - P.IVA/Cod.Fis. 01279680480

E-mail: clara.silva@unifi.it

\section{(9) $(1)$}

This work is licensed under a Creative Commons Attribution-NonCommercial 4.0 International (CC BY-NC 4.0) 